\title{
STRONG INCOMPACTNESS FOR SOME NON-PERFECT RINGS
}

\author{
JAN TRLIFAJ
}

(Communicated by Lance W. Small)

\begin{abstract}
Answering a question of Eklof and Mekler, for each regular uncountable cardinal $\kappa$, we construct a non-left-perfect ring $R_{\kappa}$ and a nonprojective strongly $\kappa$-free left ideal $I_{\kappa}$ such that $\operatorname{gen}\left(I_{\kappa}\right)=\kappa$. Moreover, if $\kappa>\aleph_{1}$, then $I_{\kappa}$ is not $\kappa$-free. As consequences, we obtain results concerning incompactness spectra of non-perfect rings.
\end{abstract}

A famous result of Kaplansky says that the structure of projective modules over an arbitrary associative ring with unit reduces to the description of the countably generated ones. This is in sharp contrast with the structure of almost free modules. Given a non-left-perfect ring $R$ and an almost free module $M$, the structure of $M$ essentially depends on the combinatorial properties of $\operatorname{gen}(M)$, the minimal cardinality of an $R$-generating subset of $M$. Here, the main split goes between singular and regular cardinals. For its study, two important notions were introduced in [EM]:

$M$ is $\kappa$-free provided there is a set, $\mathscr{C}$, of free submodules of $M$ such that: (1) $\operatorname{gen}(X)<\kappa$ for all $X \in \mathscr{C}$, (2) for each subset $A \subseteq M$ with $\operatorname{card}(A)<\kappa$ there is some $X \in \mathscr{C}$ with $A \subseteq X$, and (3) $\mathscr{C}$ is closed under unions of well-ordered chains of length $<\kappa$.

$M$ is strongly $\kappa$-free provided there is a set, $\mathscr{S}$, of free submodules of $M$ such that: (1) $\operatorname{gen}(X)<\kappa$ for all $X \in \mathscr{S}$, (2) $0 \in \mathscr{S}$, and (3) for any subset $A \subseteq M$ with $\operatorname{card}(A)<\kappa$ and any $X \in \mathscr{S}$ there is some $Y \in \mathscr{S}$ such that $X \cup A \subseteq Y$ and $Y / X$ is free.

If $\operatorname{gen}(M)=\mu$ is singular, Shelah's compactness theorem applies: $M$ is free provided $M$ is $\mu$-free ([EM, IV, Theorem 3.5]).

Let $\kappa$ be a regular uncountable cardinal. If $\operatorname{gen}(M)=\kappa$, then (in most cases) no such result holds and there is incompactness of various kinds. Of course, if $R$ is a free ideal ring, then any strongly $\kappa$-free module is $\kappa$-free. For example, this is the case when $R=\mathbb{Z}$, the ring of all integers. On the other hand, for an arbitrary ring $R$, each $\kappa^{+}$-free module is strongly $\kappa$-free ([EM, IV, Theorem 3.4]).

It was an open problem ([EM, p. 87]) whether each strongly $\kappa$-free module is $\kappa$-free. First, we obtain a positive solution for $\kappa=\aleph_{1}$ and $\operatorname{gen}(M) \leq \aleph_{1}$,

Received by the editors March 2, 1993 and, in revised form, April 6, 1993.

1991 Mathematics Subject Classification. Primary 16D40, 16E50, 03 E75.

Key words and phrases. Almost free module, non-left-perfect ring, $\kappa$-free, strongly $\kappa$-free, incompactness spectrum. 
provided either $R$ is countable or all projective modules are free. Our main result then gives a negative solution to the problem for each $\kappa>\aleph_{1}$.

More specifically, for each $\aleph_{0} \leq \lambda \leq \kappa$, we say that

$M$ is $(\kappa, \lambda)$-free provided there is a set, $\mathscr{C}$, of free submodules of $M$ such that (1) and (2) from the definition of $\kappa$-free hold, and $\left(3_{\lambda}\right) \mathscr{C}$ is closed under unions of well-ordered chains of length $<\lambda$.

Clearly, $(\kappa, \lambda)$-free implies $\left(\kappa, \lambda^{\prime}\right)$-free for all $\aleph_{0} \leq \lambda^{\prime} \leq \lambda \leq \kappa$. Moreover, $M$ is $\kappa$-free iff $M$ is $(\kappa, \kappa)$-free. In Proposition 1 , we show that any $\leq \kappa$ generated strongly $\kappa$-free module is $\left(\kappa, \aleph_{1}\right)$-free, provided either $\operatorname{card}(R)<\kappa$ or all projective modules are free. On the other hand, for each $\kappa>\aleph_{1}$, we prove that there are a non-left-perfect ring $R_{\kappa}$ and a non-projective left ideal $I_{\kappa}$ such that $\operatorname{gen}\left(I_{\kappa}\right)=\kappa, I_{\kappa}$ is strongly $\kappa$-free and $\left(\kappa, \aleph_{1}\right)$-free, but not $\left(\kappa, \aleph_{2}\right)$-free (Theorem 8).

As an immediate consequence, we obtain our

Theorem. For each regular cardinal $\kappa>\aleph_{1}$, there are a non-left-perfect ring $\boldsymbol{R}_{\kappa}$ and a non-projective strongly $\kappa$-free module $M_{\kappa}$ with $\operatorname{gen}\left(M_{\kappa}\right)=\kappa$ such that $M_{\kappa}$ is not $\kappa$-free.

Following [EM, p. 224], given a non-left-perfect ring $R$, we denote by $\operatorname{Inc}^{\prime}(R)$ the incompactness spectrum of $R$, i.e., the set of all uncountable cardinals $\lambda$ such that there is a non-projective $\lambda$-free module $M$ with $\operatorname{gen}(M) \leq \lambda$. Similarly, $\operatorname{Sinc}^{\prime}(R)$ is the strong incompactness spectrum of $R$, i.e., the set of all uncountable cardinals $\lambda$ such that there is a non-projective strongly $\lambda$-free module $M$ with $\operatorname{gen}(M) \leq \lambda$. The fundamental result of Shelah, Eklof, and Mekler ([EM, VII, Corollary 3.13]) says that

$$
\operatorname{Inc}^{\prime}(\mathbb{Z})=\operatorname{Sinc}^{\prime}(\mathbb{Z})=\bigcap_{R \in \mathscr{N}} \operatorname{Sinc}^{\prime}(R)=\bigcap_{R \in \mathscr{N}} \operatorname{Inc}^{\prime}(R),
$$

$\mathscr{N}$ being the class of all non-left-perfect rings. A recent result of Magidor and Shelah ([EM, p. 191]) shows that assuming the existence of some large cardinals, there is a model of $Z F C+G C H$ such that $\aleph_{\omega^{2}+1} \notin \operatorname{Inc}^{\prime}(\mathbb{Z})$, and a model with $\lambda \notin \operatorname{Inc}^{\prime}(\mathbb{Z})$ for all $\lambda \geq$ the first cardinal fixed point. Recall that the main open problem ([EM, p. 453, Problem 1]) is whether $\operatorname{Inc}^{\prime}(R)=\operatorname{Inc}^{\prime}(\mathbb{Z})$ for all $R \in \mathscr{N}$ (in $Z F C$ ). Though we do not solve this problem here, we have a related corollary to Theorem 8 and [EM, IV, Theorem 3.2]:

Corollary. For each regular uncountable cardinal $\kappa$, there is a non-left-perfect ring $R_{\kappa}$ such that $\kappa \in \operatorname{Sinc}^{\prime}\left(R_{\kappa}\right)$. Moreover,

(i) if $\kappa$ is weakly compact, then $\kappa \in \operatorname{Sinc}^{\prime}\left(R_{\kappa}\right) \backslash \bigcup_{R \in \mathcal{N}} \operatorname{Inc}^{\prime}(R)$,

(ii) in the first and second models of Magidor and Shelah,

$$
\operatorname{Sinc}^{\prime}\left(R_{\aleph_{\omega^{2}+1}}\right) \neq \operatorname{Sinc}^{\prime}(\mathbb{Z}) \text {, and } \operatorname{Sinc}^{\prime}\left(R_{\lambda}\right) \neq \operatorname{Sinc}^{\prime}(\mathbb{Z})
$$

for all regular $\lambda \geq$ the first cardinal fixed point, respectively.

A ring $R$ is non-left-perfect provided there is a strongly decreasing countably infinite chain of principal right ideals of $R, R$ is von Neumann regular provided each $x \in R$ has a pseudo-inverse $y \in R$ (i.e., $x y x=x$ ). A set $\left\{e_{\alpha} ; \alpha<\kappa\right\}$ is a set of orthogonal idempotents of $R$ if $0 \neq e_{\alpha}=e_{\alpha}^{2}$ and $e_{\alpha} e_{\beta}=0$ for all $\alpha, \beta<\kappa$ such that $\alpha \neq \beta$. For $r \in R$, define $\operatorname{Ann}_{R}(r)=\left\{r^{\prime} \in R ; r^{\prime} r=0\right\}$. 
Homomorphisms in module categories are written as acting on the opposite side from the scalars. The category of all (unitary left $R-$ ) modules is denoted by $R$-Mod. A system of modules $\left(M_{\alpha} ; \alpha<\kappa\right)$ is said to be a smooth chain provided $M_{0}=0, M_{\alpha} \subseteq M_{\alpha+1}$ for all $\alpha<\kappa$, and $M_{\alpha}=\bigcup_{\beta<\alpha} M_{\beta}$ for all limit ordinals $\alpha<\kappa$. A smooth chain with $M=\bigcup_{\alpha<\kappa} M_{\alpha}$ is a $\kappa$-filtration of $M$ provided $\operatorname{gen}\left(M_{\alpha}\right)<\kappa$ for all $\alpha<\kappa$.

A closed and cofinal (= unbounded) subset of a regular uncountable cardinal $\lambda$ is said to be a $c u b$. A subset $E \subseteq \lambda$ is stationary in $\lambda$ provided $E \cap C \neq \varnothing$ for each cub $C$.

For basic properties of these notions, the reader is referred to [AF], [EM], and $[\mathrm{G}]$.

We start with the case of $\lambda=\aleph_{1}$ :

Proposition 1. Let $R$ be an arbitrary ring and $\kappa$ a regular uncountable cardinal. Assume that either $\operatorname{card}(R)<\kappa$ or all projective modules are free. Then each $\leq \kappa$ generated strongly $\kappa$-free module is $\left(\kappa, \aleph_{1}\right)$-free. In particular, strongly $\aleph_{1}$-free implies $\aleph_{1}$-free provided $\operatorname{gen}(M) \leq \aleph_{1}$ and either $R$ is countable or all projective modules are free.

Proof. By [EM, IV, Lemma 1.10 and Proposition 1.11], there is a $\kappa$-filtration, $\left(M_{\alpha} ; \alpha<\kappa\right)$, of $M$ such that for all $\alpha<\beta<\kappa, M_{\alpha+1}$ and $M_{\beta+1} / M_{\alpha+1}$ are free. Denote by $\mathscr{C}$ the set of all $M_{\alpha}, \alpha<\kappa$, such that either $\alpha$ is non-limit or $\alpha$ is a limit ordinal of cofinality $\omega$. Then each element of $\mathscr{C}$ is a free module. Clearly, $\mathscr{C}$ satisfies the conditions (1), (2), and $\left(3_{\aleph_{1}}\right)$ from the definition of $\left(\kappa, \aleph_{1}\right)$-free.

Now, we fix our notation for the rest of the paper in the following Definitions 2 and 4.

Definition 2. Let $\kappa$ be a regular uncountable cardinal and $E$ a stationary subset of $\kappa$ consisting of limit ordinals. Let $K$ be a skew-field. Denote by $L$ the right linear $K$-space of dimension $\kappa$, and let $S=\operatorname{End}\left(L_{K}\right)$, i.e., $S$ is the ring of all linear transformations of $L$. Put $T=\{f \in S$; $\operatorname{rank}(f)<\kappa\}$. It is well known that $T$ is the unique maximal two-sided ideal of $S$. Put $R=S / T$.

Lemma 3. $R$ is a simple von Neumann regular ring and $R$ is not left perfect. Moreover, a module $M \in R$-Mod is projective iff $M$ is free.

Proof. Since $S$ is von Neumann regular and $T$ is maximal, $R$ is a simple von Neumann regular ring. Since $\kappa \geq \aleph_{0}, R$ contains an infinite set of orthogonal idempotents, whence $R$ is neither left nor right perfect. If $0 \neq P$ is a projective module, then $P \simeq \oplus \sum_{\alpha<\lambda} R g_{\alpha}$ for a cardinal $\lambda>0$ and non-zero idempotents $g_{\alpha} \in R, \alpha<\lambda$ ([AF, p. 300]). For each $\alpha<\lambda$, there is an idempotent $h_{\alpha} \in S$ with $R\left(h_{\alpha}+T\right)=R g_{\alpha}$. Put $H_{\alpha}=\operatorname{Ker}\left(h_{\alpha}\right)$ and $H_{\alpha}^{\prime}=\operatorname{Im}\left(h_{\alpha}\right)$. Then $L=H_{\alpha} \oplus H_{\alpha}^{\prime}$ in Mod- $K$. Since $g_{\alpha} \neq 0$, there is a $K$-isomorphism, $x_{\alpha}$, of $H_{\alpha}^{\prime}$ onto $L$. Extending $x_{\alpha}$ to $L$ by zero values on $H_{\alpha}$, we obtain $s_{\alpha} \in S$ such that $S s_{\alpha}=S h_{\alpha}$ and $\operatorname{Ann}_{S}\left(s_{\alpha}\right)=0$. Moreover, $\operatorname{Ann}_{R}\left(s_{\alpha}+T\right)=T$, whence $R g_{\alpha}=R\left(h_{\alpha}+T\right)=R\left(s_{\alpha}+T\right) \simeq R$.

Definition 4. Let $B=\left\{b_{\beta \gamma} ;(\beta, \gamma) \in \kappa \times \kappa\right\}$ be a right $K$-basis of $L$. For each $\alpha<\kappa$, define $c_{\alpha}, d_{\alpha} \in S$ by

$c_{\alpha}\left(b_{\beta \gamma}\right)=b_{\beta \gamma}$ provided $\beta \leq \alpha$ and $\gamma<\kappa$, and $c_{\alpha}\left(b_{\beta \gamma}\right)=0$ otherwise, and by 
$d_{\alpha}\left(b_{\alpha \gamma}\right)=b_{\alpha \gamma}$ provided $\gamma<\kappa$, and $d_{\alpha}\left(b_{\beta \gamma}\right)=0$ otherwise.

Put $e_{\alpha}=c_{\alpha}+T$ and $f_{\alpha}=d_{\alpha}+T, \alpha<\kappa$. Clearly, $\left\{f_{\alpha} ; \alpha<\kappa\right\}$ is a set of orthogonal idempotents of $R$, and $e_{\alpha}, \alpha<\kappa$, are idempotents in $R$ such that $R f_{0}=R e_{0}, R f_{\alpha} \subseteq R e_{\alpha} \subset R e_{\beta}$, and $R e_{\alpha} \cap R f_{\beta}=0$ whenever $\alpha<\beta<\kappa$.

Define a system of left ideals $\left(I_{\alpha} ; \alpha<\kappa\right)$ as follows: $I_{0}=0, I_{\alpha+1}=R e_{\alpha}$ provided $\alpha \in E, I_{\alpha+1}=I_{\alpha}+R f_{\alpha}$ provided $\alpha \notin E$, and $I_{\alpha}=\bigcup_{\beta<\alpha} I_{\beta}$ provided $\alpha$ is a limit ordinal. Finally, put $I=\bigcup_{\alpha<\kappa} I_{\alpha}$.

Lemma 5. $I$ is a non-projective left ideal of $R, \operatorname{gen}(I)=\kappa$, and $\left(I_{\alpha} ; \alpha<\kappa\right)$ is a $\kappa$-filtration of $I$.

Proof. Clearly, $I_{\alpha+1} \subseteq R e_{\alpha}$ for all $\alpha<\kappa$, and $\left(I_{\alpha} ; \alpha<\kappa\right)$ is a smooth chain of left ideals of $R$. Hence, $I_{\alpha+1}=I_{\alpha} \oplus R f_{\alpha}$ for all $\alpha \notin E$, the chain is strictly increasing, and $\operatorname{gen}(I)=\kappa$. Moreover, $\operatorname{gen}\left(I_{\alpha}\right) \leq \operatorname{card}(\alpha)<\kappa$, whence $\left(I_{\alpha} ; \alpha<\kappa\right)$ is a $\kappa$-filtration of $I$.

For all $\alpha \in E$ and $\alpha<\beta<\kappa$, we have $I_{\beta}=R e_{\alpha} \oplus\left(I_{\beta} \cap R\left(1-e_{\alpha}\right)\right)$, and $I_{\beta} / I_{\alpha} \simeq I_{\alpha+1} / I_{\alpha} \oplus\left(I_{\beta} \cap R\left(1-e_{\alpha}\right)\right)$. Since $I_{\alpha}$ is not finitely generated, $I_{\alpha+1} / I_{\alpha}=R e_{\alpha} / I_{\alpha}$ is non-projective, and so is $I_{\beta} / I_{\alpha}$.

Assume $I$ is projective. By Lemma 3, there exist left ideals $C_{\alpha} \subset I, \alpha<\kappa$, such that $C_{\alpha} \simeq R$ for all $\alpha<\kappa$, and $I=\oplus \sum_{\alpha<\kappa} C_{\alpha}$. Put $J_{\alpha}=\oplus \sum_{\beta<\alpha} C_{\beta}$, for all $\alpha<\kappa$. Then $\left(J_{\alpha} ; \alpha<\kappa\right)$ is a $\kappa$-filtration of $I$, and $C=\left\{\alpha<\kappa ; I_{\alpha}=J_{\alpha}\right\}$ is a cub. Take $\alpha \in E \cap C$ and $\beta \in E \cap C \cap D$, where $D=\{\gamma<\kappa ; \alpha<\gamma\}$ is a cub, too. Then $I_{\alpha+1} / I_{\alpha}$ is a non-projective summand of $I_{\beta} / I_{\alpha}=J_{\beta} / J_{\alpha} \simeq$ $\oplus \sum_{\alpha \leq \gamma<\beta} C_{\gamma}$, a contradiction.

As pointed out by the referee, the proof of the non-projectivity of $I$ can also be accomplished in analogy to the proof of [EM, VII, Corollary 3.13]. Hence, the fact that all projective modules are free is not essential here.

Lemma 6. (i) Let $\alpha=0$ or $\alpha \in E$, and put $\alpha^{\prime}=\min \{\beta \in E ; \alpha<\beta\}$. Then $I_{\gamma}$ and $I_{\delta} / I_{\gamma}$ are free whenever $\alpha<\gamma<\delta<\alpha^{\prime}$.

(ii) If $\gamma$ is a limit ordinal and $\gamma<\delta<\kappa$ is such that there exists $\alpha \in E$ with $\gamma<\alpha<\delta$, then $I_{\delta} / I_{\gamma}$ is not projective.

(iii) Let $\alpha=0$ or $\alpha \in E$, and let $0<\nu<\aleph_{1}$. Then $I_{\alpha+\nu}$ is free.

(iv) If $\aleph_{0}<\lambda<\kappa, \lambda$ is a regular cardinal, and $E \cap \lambda$ is stationary in $\lambda$, then $I_{\lambda}$ is not projective.

Proof. (i) By induction on $\gamma$ satisfying $\alpha+1 \leq \gamma<\alpha^{\prime}$, we get $I_{\gamma}=\operatorname{Re}_{\alpha} \oplus$ $\oplus \sum_{\alpha<\beta<\gamma} R f_{\beta}$. Hence, $I_{\delta} / I_{\gamma} \simeq \oplus \sum_{\gamma \leq \beta<\delta} R f_{\beta}$.

(ii) Since $I_{\alpha+1}=R e_{\alpha}$ is a summand of $I_{\delta}$, and $I_{\gamma}$ is not finitely generated, the module $I_{\delta} / I_{\gamma}$ has a non-projective summand isomorphic to $I_{\alpha+1} / I_{\gamma}$.

(iii) We prove the assertion by induction on $\nu$. For $\nu=1$, we have either $I_{\alpha+1}=R f_{0}$ or $I_{\alpha+1}=R e_{\alpha}$. If $I_{\alpha+\nu}$ is free, then either $I_{\alpha+\nu+1}=I_{\alpha+\nu} \oplus R f_{\alpha+\nu}$, or $I_{\alpha+\nu+1}=R e_{\alpha+\nu}$, and $I_{\alpha+\nu+1}$ is free, too. If $\nu<\aleph_{1}$ is a limit ordinal, then either (1) $\alpha+\nu=\sup _{n<\aleph_{0}}\left(\alpha_{n}\right)$, for some elements $\alpha_{n} \in E, n<\aleph_{0}$, or (2) $\sup _{\{\beta \in E, \beta<\alpha+\nu\}}(\beta)<\alpha+\nu$. In the case (1), we have $I_{\alpha+\nu}=\bigcup_{n<\aleph_{0}} R_{\alpha_{n}}$. Then $I_{\alpha+\nu}$ is a countably generated left ideal of $R$, and $I_{\alpha+\nu}$ is free by Lemma 3 and $[\mathrm{G}$, Corollary 2.15]. In the case (2), the assertion follows from the induction premise and part (i).

(iv) As in the proof of Lemma 5, $I_{\delta} / I_{\gamma}$ is non-projective for all $\gamma \in E \cap \lambda$ and $\gamma<\delta<\lambda$. Since $E \cap \lambda$ is stationary in $\lambda$, the same argument as in Lemma 5 shows that $I_{\lambda}=\bigcup_{\delta<\lambda} I_{\delta}$ is not projective. 
Lemma 7. $I$ is strongly $\kappa$-free.

Proof. Put $\mathscr{S}=\{0\} \cup\left\{I_{\alpha+1} ; \alpha \in E\right\}$. We show that $\mathscr{S}$ is the system making $I$ strongly $\kappa$-free. By Lemma 3 and Definition $4, X \simeq R$ for any $X \in \mathscr{S} \backslash\{0\}$. Since $I=\bigcup_{\alpha \in E} I_{\alpha+1}$, for any $\alpha \in E$ and any set $A$ with $\operatorname{card}(A)<\kappa$ there is some $\beta \in E$ with $I_{\alpha+1} \cup A \subseteq I_{\beta+1}$, and clearly $I_{\beta+1} / I_{\alpha+1}$ is free.

Theorem 8. $I$ is a non-projective strongly $\kappa$-free left ideal of $R$ with $\operatorname{gen}(I)=\kappa$. Moreover, $I$ is $\left(\kappa, \aleph_{1}\right)$-free. If $\kappa>\aleph_{1}$, then I is not $\left(\kappa, \aleph_{2}\right)$-free.

Proof. The first assertion follows from Lemmas 5 and 7. By Proposition 1 and Lemma $3, I$ is $\left(\kappa, \aleph_{1}\right)$-free.

Proving indirectly, assume $\kappa>\aleph_{1}$ and $I$ is $\left(\kappa, \aleph_{2}\right)$-free. Let $\mathscr{C}$ be the system appearing in the definition of $\left(\kappa, \aleph_{2}\right)$-free. Take $J_{0} \in \mathscr{C}$. By the premise, there is some $\alpha_{0} \in E$ with $J_{0} \subseteq \operatorname{Re}_{\alpha_{0}}$. If $\beta<\aleph_{1}$ is limit, we take $J_{\beta}=\bigcup_{\gamma<\beta} J_{\gamma}$ and $\alpha_{\beta} \in E$ such that $J_{\beta} \subseteq R e_{\alpha_{\beta}}$. If $\beta<\aleph_{1}$ and $J_{0} \subseteq R e_{\alpha_{0}} \subseteq$ $\cdots \subseteq J_{\beta} \subseteq \operatorname{Re}_{\alpha_{\beta}}$, we take $J_{\beta+1} \in \mathscr{C}$ and $\alpha_{\beta+1} \in E$ such that $\operatorname{Re}_{\alpha_{\beta}} \subset J_{\beta+1} \subseteq$ $\operatorname{Re}_{\alpha_{\beta+1}}$. Put $J=\bigcup_{\beta<\aleph_{1}} J_{\beta}=\bigcup_{\beta<\aleph_{1}} \operatorname{Re}_{\alpha_{\beta}}$. By the construction, $\operatorname{gen}(J)=\aleph_{1}$. By $\left(3_{\aleph_{2}}\right), J \in \mathscr{C}$, and there exist submodules $C_{\gamma}, \gamma<\aleph_{1}$, of $J$ such that $C_{\gamma} \simeq R, \gamma<\aleph_{1}$, and $J=\oplus \sum_{\gamma<\aleph_{1}} C_{\gamma}$. Then there is some $\beta<\aleph_{1}$ such that $\oplus \sum_{\gamma<\kappa_{0}} C_{\gamma} \subset R e_{\alpha_{\beta}} \subset \oplus \sum_{\gamma<\kappa_{1}} C_{\gamma}$. Since $\oplus \sum_{\gamma<\kappa_{1}} C_{\gamma}=\operatorname{Re}_{\alpha_{\beta}} \oplus X$, where $X=$ $\left(\oplus \sum_{\gamma<\aleph_{1}} C_{\gamma}\right) \cap R\left(1-e_{\alpha_{\beta}}\right)$, we have $\oplus \sum_{\aleph_{0} \leq \gamma<\aleph_{1}} C_{\gamma} \simeq\left(\operatorname{Re}_{\alpha_{\beta}} / \oplus \sum_{\gamma<\aleph_{0}} C_{\gamma}\right) \oplus X$, the first summand being non-projective, a contradiction.

\section{REFERENCES}

[AF] F. W. Anderson and K. R. Fuller, Rings and categories of modules, Springer, New York, 1974.

[EM] P. C. Eklof and A. H. Mekler, Almost free modules, North-Holland, New York, 1990.

[G] K. R. Goodearl, Von Neumann regular rings, Pitman, London, 1979.

Department of Algebra, Faculty of Mathematics and Physics, Charles University, Sokolovska 83, 18600 Prague 8, The Czech Republic

E-mail address: trlifaj@karlin.mff.cuni.cz 